\title{
ANÁLISE ESPACIAL DA MORTALIDADE INFANTIL E SUAS RELAÇÕES SÓCIO-AMBIEN- TAIS NA ÁREA URBANA DE RIO CLARO, SP, BR
}

\section{Spatial analysis of infant mortality and its relationship to socio environmental in urban area of Rio Claro, SP, BR}

\author{
Maria Aparecida Vedovato \\ Doutoranda em Saúde Coletiva/Epidemiologia na Faculdade de Ciências Médicas/UNICAMP \\ Campinas/SP - Brasil \\ vedovat@fcm.unicamp.br \\ Roberto Wagner Lourenço \\ Pós-Graduação da Faculdade de Ciências Médicas de Campinas (UNICAMP) \\ Campinas/SP - Brasil \\ robertow@sorocaba.unesp.br \\ Maria Rita Donalisio \\ Pós-doutorado na Universidade de Kansas. \\ Campinas/SP - Brasil \\ donalisi@fcm.unicamp.br
}

Artigo recebido para publicação em 17/08/2010 e aceito para publicação em 30/09/2011.

Este trabalho obteve apoio da FAPESP (Processo n07/53640-6) na forma de bolsa de Mestrado.

RESUMO: $\quad$ Objetivo: O objetivo deste trabalho foi analisar a distribuição espacial dos coeficientes de mortalidade infantil (CMI), de mortalidade neonatal (CMNN) e de natimortalidade (CNM) e de indicadores sócio-ambientais em Unidade de Atendimento à Saúde (UAS) na área urbana de Rio Claro, SP.

Métodos: Estudo ecológico com aplicação de técnicas de análise estatística e de geoprocessamento por meio da construção de banco de dados utilizando Sistemas de Informações Geográficas e registros de mortalidade neonatal, mortalidade infantil e de natimortos, bem como os registros dos nascimentos do periodo de 2000 a 2007 e a partir dessas informações foram construídos os respectivos coeficientes de mortalidade analisados por áreas de abrangência relacionadas às unidades de atendimento da saúde. Também foram construidos indicadores de qualidade sócio-ambiental com base nas informações das condições de coleta de lixo, condições sanitárias, abastecimento de águas e das informações relacionadas às condições de escolaridade, renda e natureza da propriedade do imóvel, baseado em informações do Censo, IBGE 2000.

Resultados: As correlações mais importantes foram verificadas entre o coeficiente de natimortalidade e o índice de qualidade sócio ambiental (30\%), a mortalidade infantil apresentou $12 \%$ e a mortalidade neonatal apresentou correlação negativa. Os resultados obtidos sugerem que provavelmente existam outros fatores como geneticidade, estilo de vida e serviços de saúde influenciando na mortalidade infantil. Conclusões: Espera-se com os resultados obtidos poder auxiliar no entendimento de determinantes de 
Análise espacial da mortalidade infantil e suas relações sócio-ambientais na área urbana de Rio Claro, SP, BR Maria Aparecida Vedovato, Roberto Wagner Lourenço, Maria Rita Donalisio

agravos à saúde e ajudar na implantação de programas de gestão e planejamento de diversos setores administrativos em áreas urbanas, principalmente o setor de saúde.

Palavras-chave: Mortalidade infantil. Epidemiologia. Qualidade ambiental. Ciência da Informação.

ABSTRACT: Objectives: To analyze the spatial distribution of child mortality coefficients (CMI), recently born mortality $(C M N N)$ and stillbirths $(C N M)$, likewise socio-environmental indicators within urban areas in Public Health Units (UAS) of Rio Claro, SP.

Methods: Ecological study with statistics analysis and geoprocessing application through data bank development using Geographic Information System tools, recently born mortality, child mortality and stillbirths, as well as the records of births in the period of 2000 to 2007. From those information, obtain the respective coefficients of mortality analyzed by Public Health Units cover. In addition, socio-environmental indicators had been acquired based on information such as trash collection conditions, sanitary setting, water supply and from scholarship information, finance and housing property category, based on Censo information from Instituto Brasileiro de Geografia e Estatisca (IBGE), 2000.

Results: The most significant correlation was verified between the stillbirth and socio-environmental quality index (30\%). Child mortality reveled $12 \%$ and recently born mortality exhibited negative correlation. The obtained results suggested there are other factors such as genetically-related, life style and health services influencing child mortality.

Conclusions: The obtained results are expected to help in the understanding of health hazards determining factors and assist on the implementation of management programs from administrative sectors in urban areas, specially the public health field.

Keywords: Child Mortality. Public health. Life quality. Information Science.

\section{INTRODUÇÃO}

A Organização das Nações Unidas (ONU), desde sua criação na década de 1940, está empenhada em melhorar o nível de vida das populações seja de ordem econômica, social, cultural ou de caráter humanitário (OLIVEIRA, 2002). A mortalidade infantil é um indicador que está relacionado às situações socioeconômicas e ambientais, tais como condições de saneamento básico, nutrição, assistência médica, condições de gestação e parto e acesso e qualidade da assistência pré-natal e perinatal, que por sua vez possuem estreita relação com o espaço geográfico (VERMELHO et al., 2006) .O coeficiente de mortalidade infantil (CMI) é considerado um dos mais sensíveis indicadores de transformações sociais, sendo que estudo sobre seu comportamento espacial colabora para a diminuição de seus níveis (BEZERRA et al., 2007).
As relações entre a localização espacial e os eventos de saúde, estudadas na dimensão temporal, são bons exemplos de estudos no campo da saúde capazes de avaliar o impacto de condições ambientais adversas sobre a saúde das pessoas. Quanto à mortalidade infantil é fundamental a compreensão destas relações, além do acesso e eficácia do sistema de atenção à saúde (BEZERRA et al., 2007; GUIMARÃES et al., 2003). Desta forma as dimensões espaciais e temporais permitem identificar com mais clareza contextos demográficos, sociais, econômicos, culturais além do ambiental ligados ao meio físico. (SCHWARZ; MARCUS, 1990).

A incorporação da categoria espaço nos estudos de saúde permite estabelecer diferenciações entre regiões conforme características que as distingam e também discutir diferenças entre estas regiões e sua relação com a estrutura espacial na qual estão inseridas (GUIMARÃES et al., 2003). 
As técnicas de geoprocessamento permitem a construção de Sistemas de Informações Geográficas (SIG) por meio da incorporação da localização e do tempo aos estudos de eventos de saúde, facilitando a identificação de padrões de distribuição espacial em diferentes unidades territoriais possibilitando a representação de uma totalidade de elementos sociais no espaço. Desta forma, o geoprocessamento permite uma rápida apresentação, superposição e interação através de mapas com qualidade e fidelidade dos dados (CUNHA; QUINTANILHA, 2003; BARCELLOS; BASTOS, 1996).

Sendo assim, estudar a distribuição espacial da mortalidade infantil e verificar a sua relação com o território em áreas urbanas deve contribuir com avanços no entendimento de determinantes de agravos à saúde e deve colaborar com a implantação de políticas de saúde que podem auxiliar na redução da mortalidade infantil.

O objetivo deste trabalho foi analisar a distribuição espacial da mortalidade infantil (MI) através da análise dos coeficientes de mortalidade infantil (CMI), de mortalidade neonatal (CMNN) e de natimortalidade (CNM) e de indicadores sócio-ambientais em Unidade de Atendimento à Saúde (UAS) na área urbana de Rio Claro, SP.

\section{MÉTODOS}

Estudo ecológico exploratório em Unidade de Atendimento à Saúde (UAS) sobre a distribuição espacial da mortalidade infantil no período de 2000 a 2007.

O local de estudo foi o município de Rio Claro, localizado na porção centro-leste do estado de São Paulo, distante $173 \mathrm{~km}$ da capital integrando a região administrativa de Campinas, segundo pólo industrial do Estado em constante expansão econômica. A população em 2007 foi estimada em 185.421 habitantes distribuídos em uma área territorial de $498 \mathrm{Km}^{2}$, sendo 99\% residente na área urbana (IBGE, 2000). Segundo o Departamento Autônomo de Água e Esgoto da cidade (DAE), a totalidade dos moradores da área urbana têm água encanada e tratada; $99,4 \%$ do esgoto coletado com $30 \%$ tratado. A coleta de lixo é realizada em 95,3\% dos domicílios e o índice de iluminação pública é de 98\% (PINTAUDI, 2003).

$\mathrm{Na}$ área da saúde, o município possui 10 equipes de Estratégia de Saúde da Família, em oito Unidades de Saúde da Família (USF) abrangendo cerca de $20 \%$ da área territorial do município; seis Unidades Básicas de Saúde; um hospital público e outros três particulares, além do sistema de vigilância epidemiológica e sanitária (Figura 1).

Foram construídos indicadores de mortalidade referentes às áreas de abrangência relacionadas com as Unidades de Atendimento à Saúde do município. As informações foram obtidas junto ao SIM e SINASC por meio da Vigilância Epidemiológica da Fundação Municipal de Saúde de Rio Claro (VE/FMS-RC). Os Indicadores de Mortalidade de Rio Claro estudados foram:

Coeficiente de Mortalidade Infantil:

$C M I=\frac{\text { Número de óbitos de menores de } 1 \text { ano, na área } A \text {, no ano } T}{\text { Número de nascidos vivos, na área } A \text {, no ano } T} \times 1000$

Coeficiente de Natimortalidade:

$C N M=\frac{\text { Número de nascidos mortos, na área } A \text {, no tempo } T}{\text { Número de nascidos vivos }+ \text { nascidos mortos na área } A \text {, no tempo } T} \times 1000$

Coeficiente de Mortalidade Neonatal:

$C M N N=\frac{\text { Número } \text { de óbitos de menores de } 28 \text { dias na área } A \text {, no ano } T}{\text { Número de nascidos vivos, na área } A \text {, no ano } T} \times 1000$ 
Análise espacial da mortalidade infantil e suas relações sócio-ambientais na área urbana de Rio Claro, SP, BR Maria Aparecida Vedovato, Roberto Wagner Lourenço, Maria Rita Donalisio

Figura : Localização do município de Rio Claro

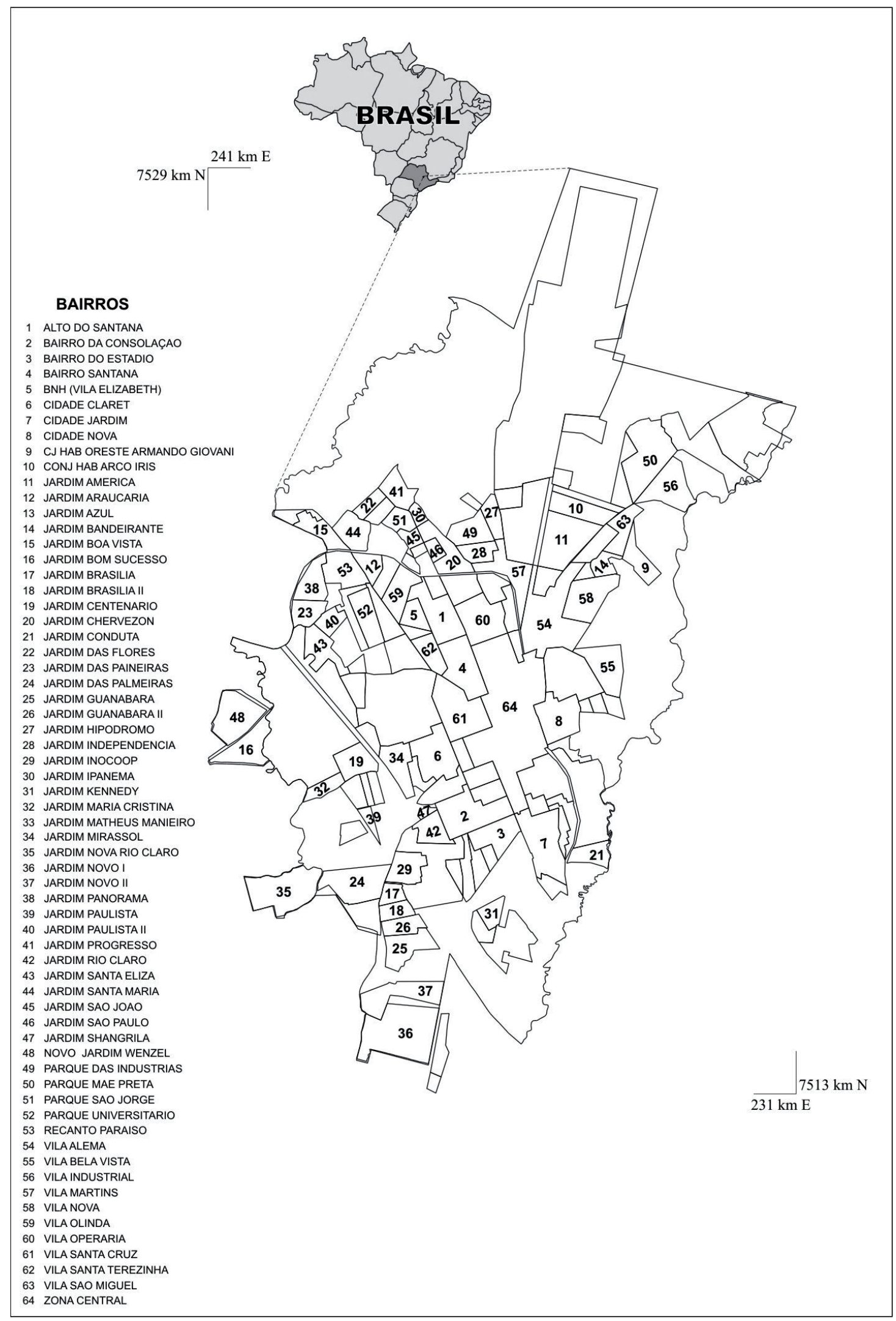

Soc. \& Nat., Uberlândia, ano 23 n. 3, 435-452, set/dez. 2011 
Os Índices de Qualidade Sócio Ambiental (IQSA) utilizados neste trabalho foram construídos por meio de informações sócio-ambientais que refletem as condições materiais ou sociais com a finalidade de identificar estes índices na área de estudo (AKERMAN; CAMPANARIO, 1996). Foram utilizadas as informações do Censo 2000 referentes aos 197 setores censitários da área de estudo classificados em dois grupos de indicadores: um grupo formado pelas informações referentes às condições das moradias composto pelo tipo de coleta de lixo (LIX), condições sanitárias (coleta esgoto) (SAN) e abastecimento de água das moradias (ABA) sendo denominado de Índice da Qualidade das Moradias (IQM); e outro grupo formado pelas informações relacionadas às condições de escolaridade (ESC), renda (REN) e natureza da propriedade do imóvel (NPI) para próprio, alugado ou cedido sendo denominado Índice da Inserção Social (IIS). Aos grupos de informações sócio-ambientais foram atribuídos pesos de acordo com sua importância sócio-ambiental (Tabela 1).

TABELA 1 Atribuição de pesos para os indicadores

\begin{tabular}{c|l|c|c|c|c}
\hline \multirow{2}{*}{ Indicadores } & \multicolumn{1}{|c|}{ Variáveis } & \multicolumn{3}{c}{ Condições (notas atribuídas) } \\
\cline { 3 - 6 } & \multicolumn{1}{|c|}{ Ótimo } & Bom & Regular & Ruim \\
\hline \multirow{4}{*}{ IQM } & $\begin{array}{l}\text { Abastecimento de água } \\
\text { (ABA) }\end{array}$ & 1,00 & 0,50 & 0,25 & 00 \\
\cline { 2 - 6 } & Condição sanitária (SAN) & 1,00 & 0,50 & 0,25 & 00 \\
\cline { 2 - 6 } & Destino do lixo (LIX) & 1,00 & 0,50 & 0,25 & 00 \\
\hline \multirow{4}{*}{ IIS de ocupação } & 1,00 & 0,75 & 0,50 & 0,25 \\
& $\begin{array}{l}\text { Condição } \\
\text { (COD) }\end{array}$ & 1,00 & 0,75 & 0,50 & 0,25 \\
\cline { 2 - 6 } & Renda (REN) & 1,00 & 0,75 & 0,50 & 0,25 \\
\cline { 2 - 6 } & Escolaridade (ESC) & & & & \\
\hline
\end{tabular}

Após a atribuição dos pesos os grupos de informações sócio-ambientais foram compostos para formarem os indicadores IQM e IIS por meio de média ponderada de acordo com as equações 4 e 5 apresentadas a seguir:

$$
\begin{gathered}
I Q M_{n}=\left(0,2 * \mathrm{ABA}_{\mathrm{n}}\right)+\left(0,5 * S A N_{n}\right)+\left(0,3 * L I X_{n}\right) \\
I I S_{n}=\left(0,2 * N P I_{n}\right)+\left(0,3 * R E N_{n}\right)+\left(0,5 * E S C_{n}\right)
\end{gathered}
$$


Análise espacial da mortalidade infantil e suas relações sócio-ambientais na área urbana de Rio Claro, SP, BR Maria Aparecida Vedovato, Roberto Wagner Lourenço, Maria Rita Donalisio

Em seguida os indicadores IQM e IIS foram utilizados para comporem o índice de qualidade sócio-ambiental (IQSA), sendo novamente aplicada a média ponderada em seu cálculo de acordo com a expressão 6 a seguir:

$$
I Q S A_{n}=\left(0,4 * I Q M_{n}\right)+\left(0,6 * I I S_{n}\right)
$$

Finalmente os valores de IQSA foram escalonados em notas variando quantitativamente de 1 a 4 , sendo que a nota 4 foi relacionada qualitativamente às áreas com os piores indicadores de IQSA e a nota 1 foi relacionada qualitativamente às áreas com os melhores indicadores de IQSA (Tabela 2).

Tabela 2: Peso Final

\begin{tabular}{ccc}
\hline IQSA & QUANTITATIVO & QUALITATIVO \\
\hline $\mathbf{( 0 , 6 5 - 1 , 0 0 )}$ & 1 & ÓTIMO \\
$\mathbf{( 0 , 5 0 - 0 , 6 5 )}$ & 2 & BOM \\
$(\mathbf{0 , 2 5 - 0 , 5 0 )}$ & 3 & REGULAR \\
$(\mathbf{0 , 2 5 )}$ & 4 & RUIM \\
\hline
\end{tabular}

Construção do Sistema de Informações Geográficas

Os indicadores de mortalidade e os sócio-ambientais foram georeferenciadas na base cartográfica de Rio Claro, permitindo a elaboração de mapas temáticos da distribuição espacial do Índice de Qualidade Sócio Ambiental (IQSA), e do Coeficiente de Mortalidade Infantil, Coeficiente de Mortalidade Neonatal e Coeficiente de Natimortalidade na área de estudo no período estudado.

Foi utilizada a Carta Cadastral do município de Rio Claro, do ano 2000, para delimitar a área de estudo, retificada no AutoCad Map 2004 (Autodesk, 2000) de acordo com levantamentos recentes disponibilizados pelo setor de planejamento da prefeitura e trabalho de campo utilizando-se aparelho de Global Positioning System-GPS do modelo Garmin III Plus (Garmin Inc., 2003).

As unidades de estudo foram as áreas de abrangência relacionadas com as Unidades de Atendimento à Saúde do município. O município possui 15 unidades de atendimento a população distribuída da seguinte forma: 05 Unidade Básica de Saúde
(UBS), 07 Unidade de Saúde da Família (USF) e 3 Pronto Atendimentos 24 horas (PA). Nessas unidades são priorizados atendimentos clínicos, ginecológico e pediátrico e alguns atendimentos de emergências. Os atendimentos especializados são encaminhados ao Centro de Análise e Apoio Diagnóstico (CEAD) e os casos mais graves que necessitam de cirurgias e internações são encaminhados aos principais hospitais do município da cidade.

As áreas de abrangência relacionadas com as Unidades de Atendimento à Saúde (UAS) da área de estudo foram construídas segundo critérios que consideraram a abrangência do endereço das residências dos usuários e os obstáculos físicos dos trajetos, tais como rodovias, ferrovias e hidrografia. Foram definidas 13 áreas de abrangência das 15 Unidades de Atendimento à Saúde, tendo em vista que as unidades 2 e 3 terem sido agrupadas em uma única unidade de abrangência (Figura 2a-b). 
Figura 2-a: Obstáculos na área urbana

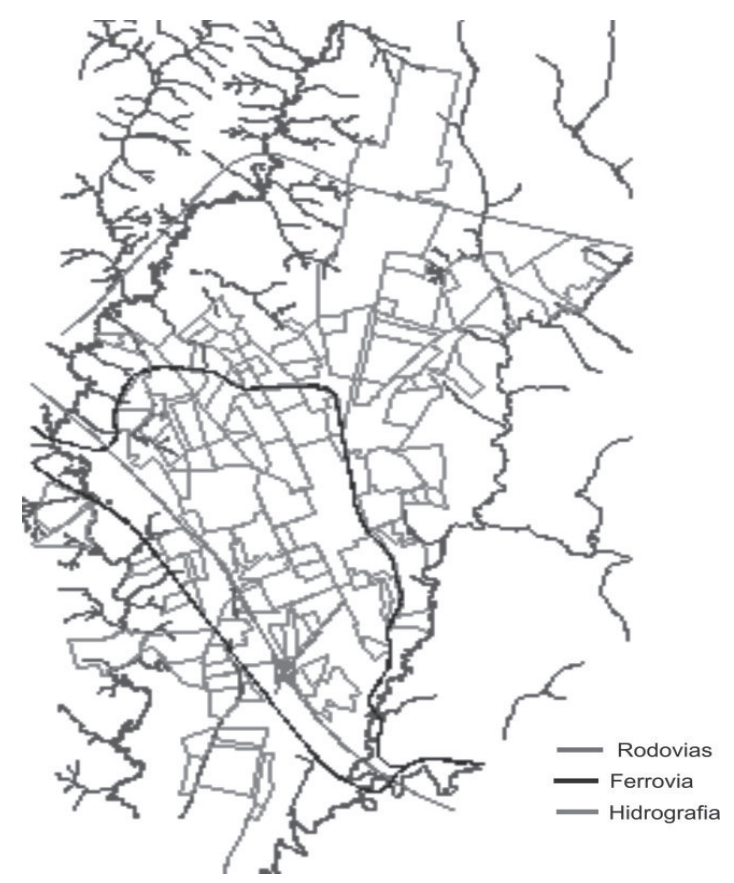

Figura 2-b: Unidades de Atendimento à Saúde (UAS)



Soc. \& Nat., Uberlândia, ano 23 n. 3, 435-452, set/dez. 2011 
Essas UAS foram utilizadas para os cálculos e análises dos indicadores de mortalidade infantil. A espacialização dos indicadores por UAS permite uma melhor compreensão destes indicadores por se tratar de escala local que considera aspectos funcionais, tais como construções e estruturais viárias, bem como aspectos do meio ambiente, como rios, lagos etc.

A espacialização das variáveis MI e dos indicadores sócio-ambientais foi feita utilizando-se

um Sistema de Informações Geográficas (SIG) por meio de procedimento georrelacional entre tabelas anuais de CMI, CMNN e CNM com as estruturas gráficas (polígonos) conectados espacialmente por códigos de mesma identificação. Este procedimento é chamado de geocodificação para variáveis associadas a um sistema de coordenadas geográficas. Nesta etapa foram utilizados os programas AutoCad Map (2004) para digitalização dos polígonos e ArcGis 9.2 para conectar as tabelas.

Em seguida foi executado um procedimento de interpolação dos valores de CMI, CMNN, CNM e dos IQSA baseado em valores de amostras vizinhas de uma mesma área proporcional ao inverso às respectivas distâncias elevadas a uma potência (LANDIM, 2003), gerando um modelo de distribuição espacial contínuo de acordo com a seguinte expressão:

$$
Z_{p}=\frac{\sum_{i=1}^{n} \frac{Z_{i}}{h_{i j}^{\beta}}}{\sum_{i=1}^{n} \frac{1}{h_{i j}^{\beta}}}
$$

$\mathrm{Z}_{p}$ é valor estimado dos CMI, CMNN, CNM e dos IQSA associado ao centróide do polígono de cada uma das diferentes UAS;

$Z i$ é o valor do resíduo entre o ponto amostrado mais próximo vizinho ao valor estimado

hij é a distância entre o valor estimado e o valor amostrado;

$\beta$ é o expoente de ponderação (peso)

$n$ é número de pontos amostrados utilizados para estimar cada valor.
Nesta etapa foram utilizados os programas AutoCad Map (2004) para digitalização dos polígonos, Surfer 8.0 para a construção da superfície interpolada dos CMI para o período total e do IQSA.

Correlação linear dos CMI e IQSA

Foram realizadas análises estatísticas para verificação da correlação entre os diferentes coeficientes de MI para o período total de estudo e o IQSA através do modelo de Correlação do Produto do Momento de Pearson. Os dados de IQSA foram tratados como independentes e os diferentes coeficientes de MI como dependentes. Para essa análise utilizou-se o Teste $t$-Student com $n$ - 2 graus de liberdade e $\alpha=5 \%$ (NETO, 2002).

Para elaboração do banco de dados e análises estatísticas foram utilizados processadores de tabelas Excel, Access (Microsoft, 2007), para modelagem e análise espacial foram utilizados os programas , Idrisi 32 (Clark Labs, 2001) e Surfer 8.0 (Golden Software, 2002).

O estudo foi aprovado pelo Comitê de Ética da Faculdade de Ciências Médicas/UNICAMP.

\section{RESULTADOS}

\section{Perfil da MI na área de estudo}

O Coeficiente de Mortalidade Infantil do município foi de 2,5 por 1000, o Coeficiente de Mortalidade Neonatal de 8,4 por 1000 e o Coeficiente de Natimortalidade de 6,4 por 1000 nascidos vivos no período estudado. O resultado do perfil epidemiológico da mortalidade infantil é apresentado na Tabela 3 
Tabela 3: Perfil Epidemiológico da Mortalidade Infantil

\begin{tabular}{|c|c|}
\hline Nascimentos & 15108 registros \\
\hline Óbito infantil & $\begin{array}{l}165 \text { registros } \\
96 \text { sexo masc. }(58,18 \%) \\
69 \text { sexo fem. }(41,82 \%) \\
106 \text { óbito neo-precoce }(64,24 \%) \\
21 \text { óbito neo-tardio }(12,73 \%) \\
38 \text { óbito infantil }(23,03 \%)\end{array}$ \\
\hline $\begin{array}{l}\text { Peso ao nascer } \\
\text { (gramas) }\end{array}$ & $\begin{array}{l}\text { Mínimo } 420 \\
\text { Máximo } 4100 \\
\text { Média } 1706.59 \\
\text { Desvio padrão } 1012,42\end{array}$ \\
\hline $\begin{array}{l}\text { Idade materna } \\
\text { (anos) }\end{array}$ & $\begin{array}{l}\text { Mínimo } 14 \\
\text { Máximo } 44 \\
\text { Média } 26,56 \\
\text { Desvio padrão } 6,94\end{array}$ \\
\hline $\begin{array}{l}\text { Causas básicas } \\
\text { Com maiores } \\
\text { números de } \\
\text { ocorrências }\end{array}$ & $\begin{array}{l}\text { Problemas respiratórios ao nascer: } 53 \text { casos }(32,12 \%) \\
\text { Septicemias e afecções: } 21 \text { casos }(12,72 \%) \\
\text { Malformação congênita: } 13 \text { casos }(7,87 \%) \\
\text { Imaturidade extrema: } 12(7,27 \%) \\
\text { Broncopneumonia e asma: } 11(6,66 \%)\end{array}$ \\
\hline Natimorto & $\begin{array}{l}98 \text { registros } \\
51 \text { sexo masc. }(52,04 \%) \\
47 \text { sexo fem. }(47,96 \%)\end{array}$ \\
\hline $\begin{array}{l}\text { Peso } \\
\text { (gramas) }\end{array}$ & $\begin{array}{l}\text { Mínimo } 300 \\
\text { Máximo } 4100 \\
\text { Média } 1718,52 \\
\text { Desvio padrão } 954.81\end{array}$ \\
\hline $\begin{array}{l}\text { Idade materna } \\
\text { (anos) }\end{array}$ & $\begin{array}{l}\text { Mínimo } 15 \\
\text { Máximo } 45 \\
\text { Média } 26,14 \\
\text { Desvio padrão } 6,79\end{array}$ \\
\hline $\begin{array}{l}\text { Causas básicas } \\
\text { Com maiores } \\
\text { números de } \\
\text { ocorrências }\end{array}$ & $\begin{array}{l}\text { Feto afetado por anomalias da placenta } 20 \text { casos }(20,04 \%) \\
\text { Feto afetado por transtornos maternos hipertensivos e Feto e } \\
\text { recém-nascido afetados por compressão do cordão } 16 \text { casos } \\
(16,32 \%) \\
\text { Hipóxia intra-uterina } 15 \text { casos }(15,3 \%) \\
\text { Morte fetal de causa não especificada } 13 \text { casos }(13,26 \%)\end{array}$ \\
\hline
\end{tabular}

Análise espacial dos indicadores sócio-ambientais e da MI

As Figuras 3, 4 e 5 mostram mapas de IIS, IQM, IQSA e dos indicadores de MI referentes a todo o período, elaborados considerando os limites da área urbana do município. 
Figura 3: Mapa de IIS na área urbana de Rio Claro

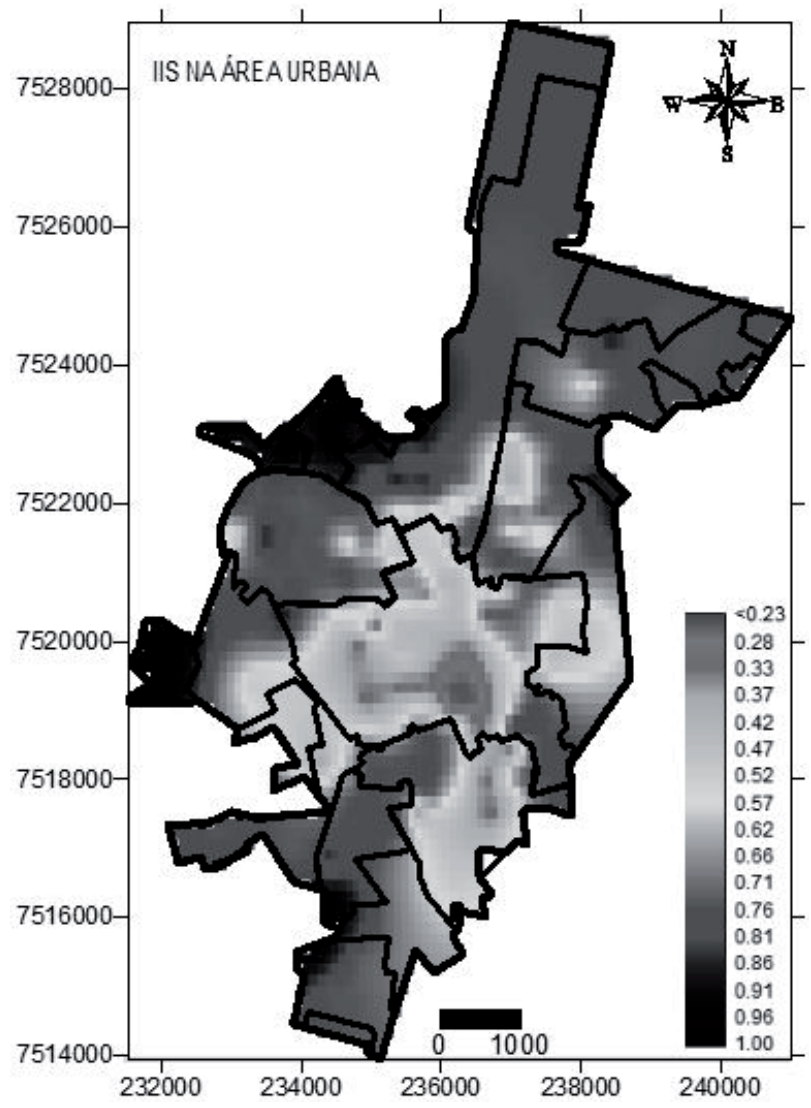

Ao analisar o mapa da distribuição espacial de IIS (Figura 3), observa-se que foi no setor leste da Zona Central que os valores de IIS se apresentaram abaixo de 0,25 , situações consideradas de baixa qualidade de IIS (RUIM) quando comparados aos valores da Tabela 2. Neste setor predomina a presença de pessoas idosas, aposentados da antiga Ferrovia Paulista S. A. (FEPASA), casarões antigos e pouco comércio.

Ainda com relação aos valores de baixa qualidade de IIS, foi observado que no setor sudeste encontrou-se valores oscilando entre 0,25 a 0,57 , classificados como REGULAR, porém podem ser considerados como de baixa qualidade de IIS. Este setor é caracterizado tanto por bairros antigos de baixo padrão como por bairros recentes de alto padrão e também um setor de indústrias.

No entanto, as maiores variações dos valores se concentraram na faixa referente à qualidade social
$\mathrm{BOM}$, ou seja, valores oscilando entre 0,50 a 0,65 . As áreas mais evidentes desta qualidade foram os setores norte-nordeste principalmente, e algumas regiões do oeste e sul-sudoeste. São áreas formadas por bairros recentes com intensa atividade industrial e comercial.

Por outro lado, as melhores condições de qualidade social, classificadas como ÓTIMO (> $0,65)$ foram encontradas principalmente nos setores noroeste-oeste, bem como em uma pequena porção do setor sul e algumas pequenas regiões isoladas do setor nordeste-leste. Estes setores caracterizam-se pela presença de bairros de médio e alto padrões e o Campus da Universidade Estadual Paulista (UNESP-RC).

O mapa da distribuição espacial do IQM é apresentado na Figura 4. 
Figura 4: Mapa de IQM na área urbana de Rio Claro

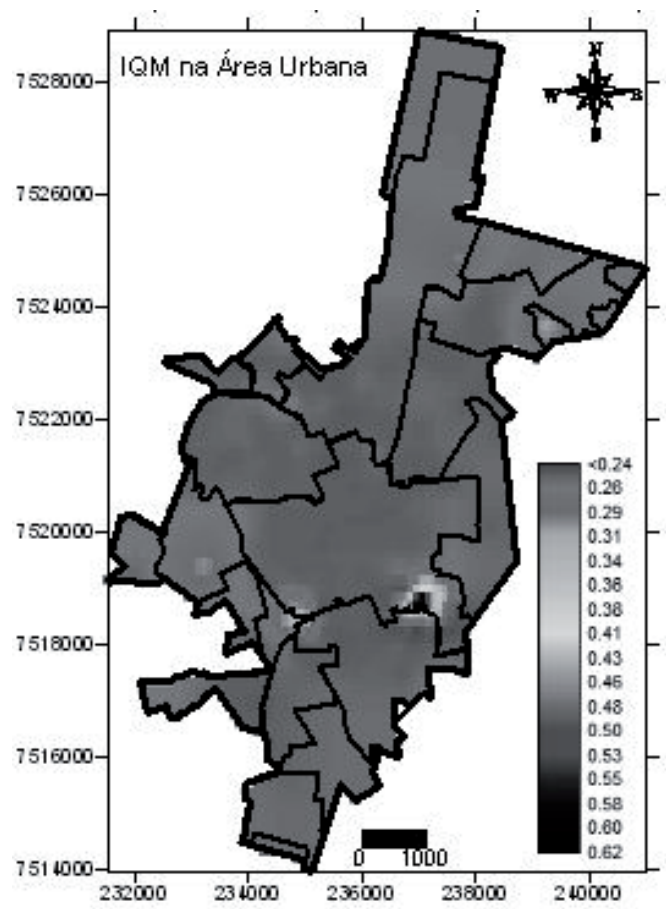

Com relação à análise da distribuição espacial dos valores do IQM no setor urbano da área de estudo (Figura 4),é possível afirmar que grande parte foi classificada com valores de qualidade de moradia RUIM (valores $<0,25$ ) (Tabela 2). Porém, apesar de pouco intensa, foi notada uma pequena variação de

qualidade de moradia de valores classificados como REGULAR entre 0,25 e 0,50 , no setor sudoeste e leste da Zona Central da área de estudo. Observam-se no setor leste, áreas com valores acima de 0,65 (figura 5).

A Figura 5 apresenta o Mapa de IQSA na área urbana da área de estudo.

Figura 5: Mapa de IQSA na área urbana de Rio Claro

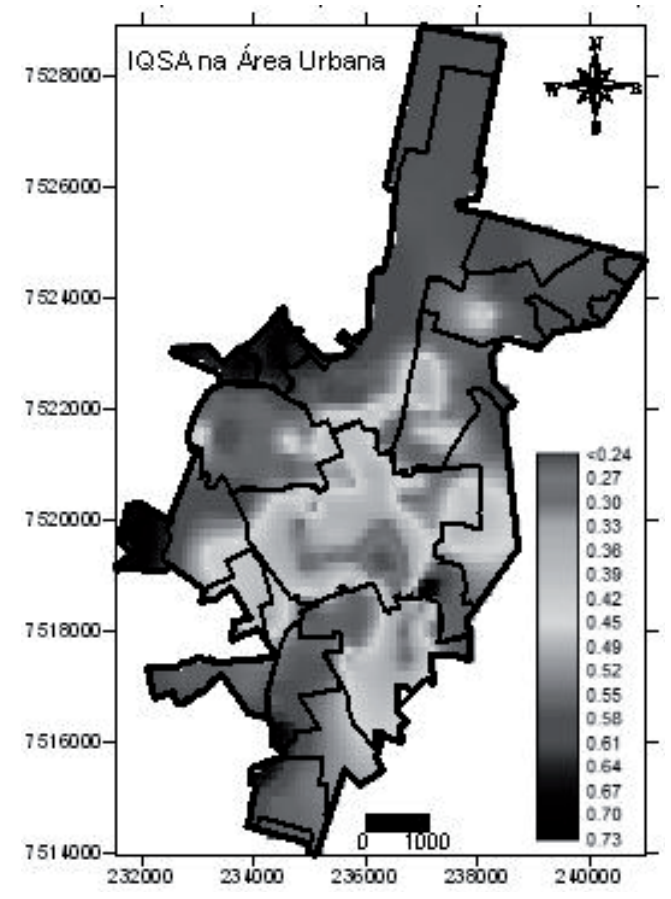


Análise espacial da mortalidade infantil e suas relações sócio-ambientais na área urbana de Rio Claro, SP, BR Maria Aparecida Vedovato, Roberto Wagner Lourenço, Maria Rita Donalisio

O índice composto IQSA da área urbana apresentou variação dos valores na faixa entre 0,24 e 0,73 (Figura 5). Observou-se que os valores que indicaram qualidade socioambiental com valores RUIM $(<25)$ foram localizados predominantemente na região sudeste do setor da zona central da área de estudo, e os valores predominantes de situação REGULAR quase que totalmente no setor central, faixas das áreas sudeste e leste (entre 0,25 a 0,50 ). Os locais com predominância de valores de IQSA BOM $(0,50$ a 0,65$)$ foram os setores norte-noroeste, algumas regiões do oeste e sudoeste. Os melhores valores predominaram nos setores noroeste-oeste e em alguns pontos do setor sudoeste-sul, com valores de IQSA oscilando acima de 0,65 .

Os mapas dos indicadores de MI de todo o período foram comparados com os indicadores sócio-ambientais (Figuras 6, 7 e 8). As faixas de valores mais altos de CMI total (entre 50 e 74 por mil nascidos vivos) foram localizadas nos setores do sudoeste-oeste da área de estudo. Nesta área o IIS está classificado próximo de valores tabelados como BOM, o IQM de valores classificados como REGULAR e o IQSA de valores classificados como BOM (Tabela 2).

Figura 6: Mapa de CMI total na área urbana de Rio Claro

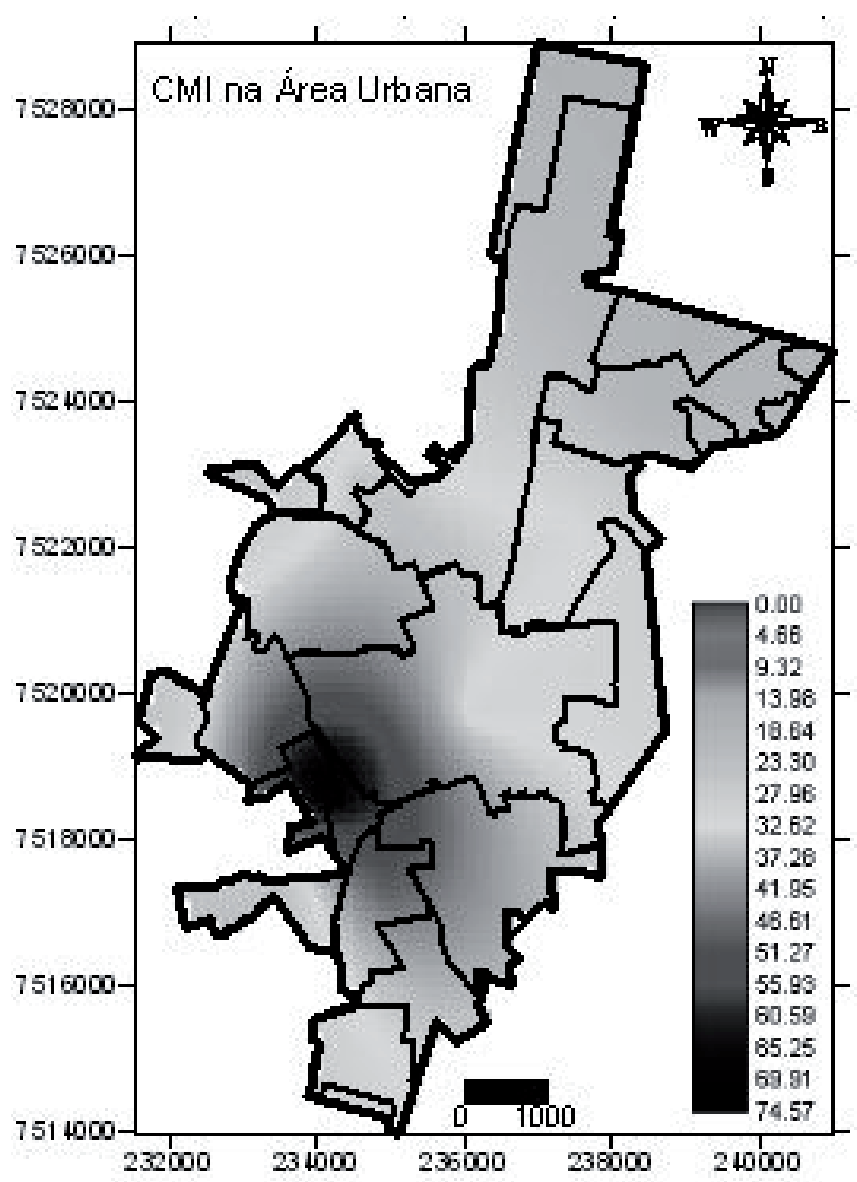

Os valores intermediários dos coeficientes de mortalidade infantil (entre 28 e 49) apresentaram neste período predominância de ocorrência espacial. Foram distribuídos por quase todos os setores, da parte central da área de estudo aos setores periféricos da área urbana. Porém nota-se tendência de diminuição destes valores da direção sudoeste para nordeste da área de estudo. Assim, os menores valores de CMI se encontraram nos setores norte-nordeste Estas áreas apresentam IIS com valores classificados como ÓTIMO (entre 0,82 e 1,00), IQM REGULAR (entre 0,25 e 0,31 ) e IQSA de REGULAR para ÓTIMO (entre 0,49 e 0,73 ) (Figura 6) 
São apresentados os resultados da distribuição espacial dos CMNN na área urbana de Rio Claro, no período de 2000 a 2007 na Figura 7. Observa-se que as faixas de valores mais altos ( 45 a 56 por mil nascidos vivos) estão localizadas nos setores sudeste-oeste
(UAS Benjamin de Castro). Nesta área o IIS está classificado próximo de valores tabelados como BOM, o IQM de valores classificados como REGULAR e o IQSA de valores classificados como BOM (Tabela 2).

Figura 7: Mapa de CMNN total na área urbana de Rio Claro

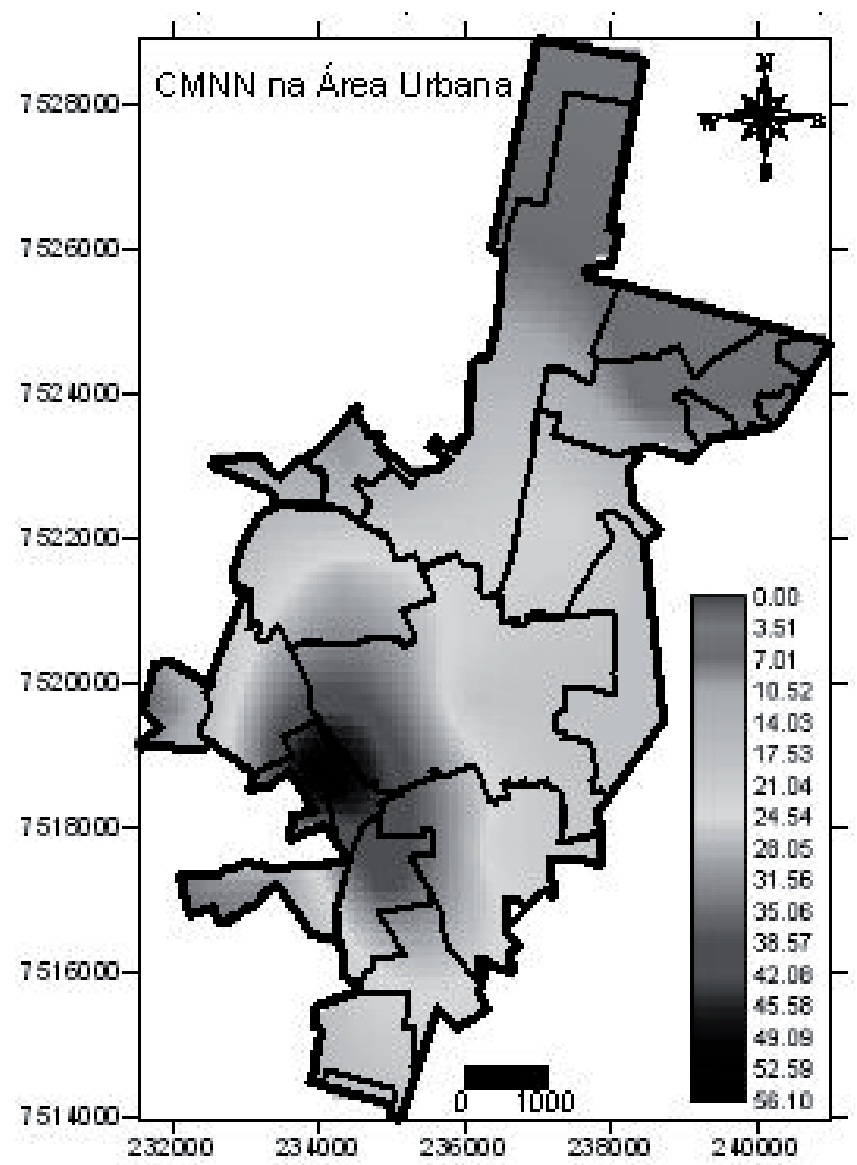

A faixa com valores mais altos vai diminuindo em direção ao sudeste, noroeste e zona central, apresentando valores entre 19 e 44 por mil nascidos vivos. Nestas áreas o IIS varia entre 0,39 e 0,91 e o IQM a variação foi pequena na faixa de valores regulares $(0,25$ e 0,31$)$, exceto a área do Bairro da Saúde no sudeste da zona central .

Os valores mais baixos de CMNN total (entre 6 e 17 por mil nascidos vivos) foram observados nos setores norte, oeste, sudoeste e noroeste. Estas áreas apresentam IIS com valores classificados como ÓTIMO (entre 0,82 e 1,00), IQM regular (entre 0.25 e 0.31 ) e IQSA de REGULAR para ÓTIMO (entre 0,49 e 0,73 ). As faixas de valores seguem de sudoeste (valor mais alto de 45 a 56) em direção aos setores sudeste, noroeste e zona central.

A figura 8 apresenta os resultados da distribuição espacial do CNM na área urbana de Rio Claro, de 2000 a 2007. A distribuição espacial do CNM total indica um comportamento diferenciado, com duas áreas de valores mais altos nas faixas entre 40 e 57 por mil nascidos vivos abrangendo os setores sudeste-oeste e noroeste. Toda a área urbana apresenta valores de CNM do período nas faixas entre 20 e 39 , exceto os extremos norte e oeste que apresentam valores nas faixas entre 2 e 19. As áreas dos extremos norte e oeste apresentam IIS com valores classificados, segundo a Tabela 2, como ÓTIMO (entre 0.82 e 1.00), IQM REGULAR (entre 0.25 e 0.31 ) e IQSA de REGULAR para ÓTIMO (entre 0.49 e 0.73 ). 
Figura 8: Mapa de CNM total na área urbana de Rio Claro

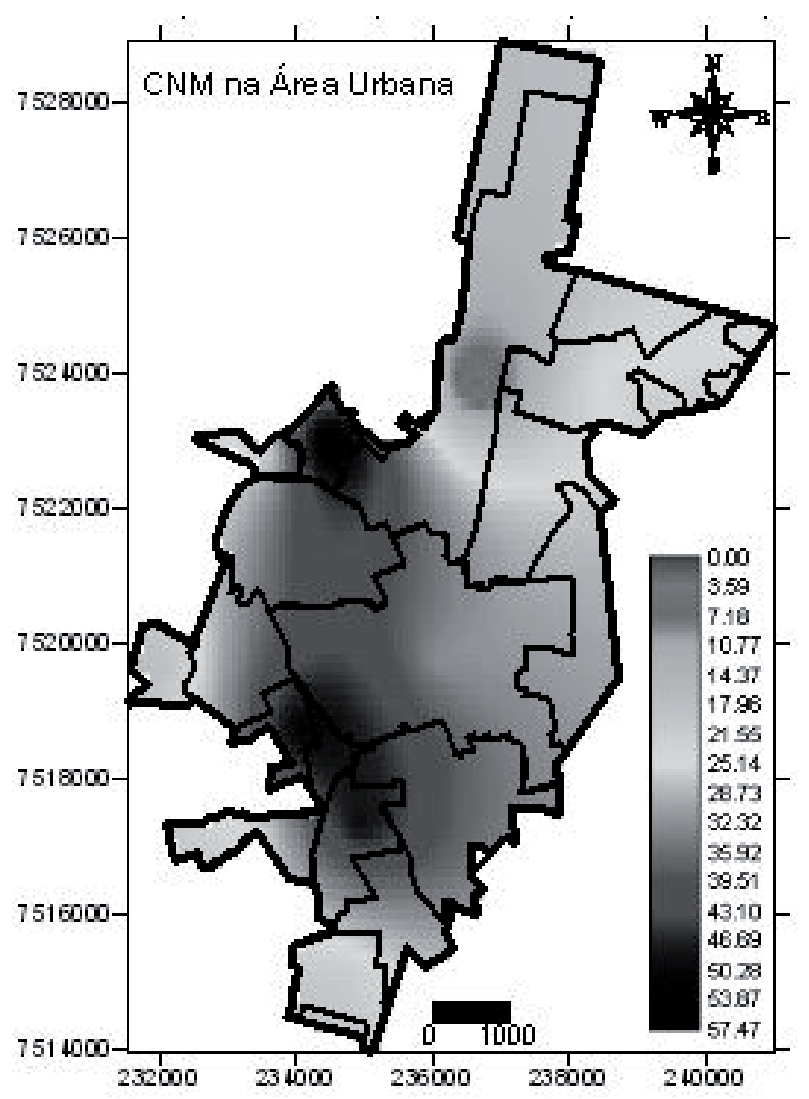

A análise estatística de IQSA e dos indicadores de MI é apresentada na tabela 3. Mostra os resultados da análise de Correlação Estatística Linear de Pearson entre o IQSA e os indicadores de MI e do teste $t$-Student para o período total. O CMNN é o indicador que apresenta correlação positiva mais importante com o IQSA, o CMI apresenta correlação positiva menos importante e o CNM apresenta correlação negativa (Tabela 4).

Tabela 4: Correlação estatística linear entre os indicadores de MI e IQSA

\begin{tabular}{c|c|c|c}
\hline IQSA & CMI & CMNN & CNM \\
\hline Coeficiente de correlação de Pearson $(\mathrm{r})$ & 0.118 & -0.0551 & 0.2733 \\
\hline Teste de Significância $(t)$ & 0,380 & -0.186 & 0.832 \\
\hline Interpretação de $t$ & Válido & Válido & Válido \\
\hline
\end{tabular}

O teste de significância dos resultados da correlação apresentado na tabela acima mostra um valor de $t$ tabelado $=2,201$ para $\mathrm{n}-2=11 \mathrm{com}$ $\alpha / 2=0,025$. Como todos os valores calculados foram inferiores ao valor tabelado, se aceita $\mathrm{H} 0$, validando a correlação. 


\section{DISCUSSÃO}

Os resultados demonstram que indicadores de mortalidade infantil se apresentam de forma heterogênea na área urbana de Rio Claro e as causas básicas da mortalidade infantil estão relacionadas principalmente com a mortalidade neonatal, predominando registros com crianças do sexo masculino, baixo peso e prematuros.

Embora os indicadores de mortalidade infantil estudados no município sejam satisfatórios, comparados à grande maioria das cidades brasileiras, os resultados menos favoráveis concentraram-se em bairros localizados a oeste e noroeste da cidade como apresentado nos mapas da distribuição espacial de CMI, CMNN, CNM. Apesar dos indicadores serem construídos com pequenos números, sujeitos a instabilidades e viéses ocasionais, a distribuição espacial dos riscos de morte no primeiro ano de vida, bem como da natimortalidade foi heterogênea na cidade. Em áreas onde houve maiores coeficientes de mortalidade infantil também se registraram maiores coeficientes de natimortalidade (oeste da área urbana).

Observou-se que os mapas do Índice de Qualidade Socioambiental (IQSA) e os mapas de CMI e CNM apresentam baixa relação entre as localizações geográficas destes índices e dos indicadores de MI. Mesmo baixa, a correlação estatística linear foi positiva entre o IQSA, CMI e CNM, já o CMNN apresentou baixa correlação linear negativa.

A mortalidade neonatal é determinada, na maioria das vezes, por malformações congênitas, por causas perinatais, como baixo peso ao nascer, prematuridade, problemas relacionados ao parto e pós-parto imediato, precariedade nos serviços de saúde de pré-natal e parto. Estas causas indicam influência das condições gerais de vida, os padrões de práticas de saúde e acesso aos serviços de saúde. Sendo assim, a maioria dos óbitos neonatais pode ser evitada por meio de condutas adequadas de controle de gravidez e atenção ao parto (BARROS et al., 2008)

Quanto aos óbitos relacionados ao período pós-neonatal, não foram encontrados registros que indicariam precárias condições ambientais em crianças com idade de 28 dias a 1 ano. Não foram registrados óbitos associados a enfermidades infeccto- -contagiosas. Predominaram registros natimortalidade e mortalidade infantil de crianças do sexo masculino. Estes resultados indicam que a área de estudo oferece boas condições de vida aos seus habitantes e sugerem evidências da existência de outros fatores decorrentes do período de gestação e pós-parto, como problemas genéticos e estilo de vida, entre outras contribuindo para as ocorrências de mortalidade infantil na área de estudo.

Estudos identificam fatores associados à mortalidade infantil no Brasil, como a precariedade da assistência pré-natal (falta de acesso e qualidade inadequada), más condições obstétricas durante e após o parto, desigualdades sócio-ambientais entre outras (BENATTI, 2003; FISCHER, et al., 2007). Assim a qualidade das práticas médico-sanitárias e o acesso aos serviços de saúde seriam os maiores determinantes na ocorrência de morbidade e mortalidade nesta faixa etária. O maior impacto das condições sócio-ambientais, saneamento e a qualidade de vida estão vinculadas a mortalidade pós-neonatal (dos 28 dias a 1 ano de vida), óbitos que não foram relevantes no município de estudo.

As técnicas de geoprocessamento e análise espacial, associadas ao manejo de dados sócio-demográficos de setores censitários disponibilizados pelo IBGE e de informações epidemiológicas da área da saúde dos municípios, podem ser utilizadas como ferramentas de gestão e planejamento no âmbito da administração pública. Contribuem para melhor compreensão da distribuição de agravos na área urbana, primeiro passo para investigação detalhada sobre os determinantes locais que explicariam melhor os riscos de adoecer e morrer na cidade. No caso de rio Claro, os indicadores menos favoráveis concentraram-se em bairros da cidade possíveis de serem identificados e priorizados pela administração local.

Em uma cidade onde não há contrastes marcantes na qualidade de vida dos moradores nem associação muito evidente entre óbitos infantis e condições ambientais, o foco da análise se dá no acesso e qualidade da assistência médico sanitária, a qual não foi abordada neste estudo.

No setor saúde, as informações epidemiológicas mapeadas podem ser instrumentos valiosos para discussão pelas equipes de saúde sobre a qualidade do 
Análise espacial da mortalidade infantil e suas relações sócio-ambientais na área urbana de Rio Claro, SP, BR Maria Aparecida Vedovato, Roberto Wagner Lourenço, Maria Rita Donalisio

pré-natal, o treinamento dos profissionais para identificar e manejar gravidez de risco, o investimento em programas de redução da gravidez na adolescência, a infra-estrutura, laboratorial, obstétrica e neonatal, hospitalar e ambulatorial, podem ser parte das ações necessárias para reduzir mortes nas primeiras semanas de vida.

\section{AGRADECIMENTOS}

A FAPESP pelo financiamento, ao setor de Vigilância Epidemiológica da Fundação Municipal de Saúde do município de Rio Claro pelo apoio e disponibilização dos dados e ao programa de Pós-graduação em Saúde Coletiva da Faculdade de Ciências Médicas/ UNICAMP.

\section{REFERÊNCIAS}

OLIVEIRA, G. B._Uma discussão sobre o conceito de desenvolvimento. Rev. FAE, Curitiba, v. 5, n. 2, p. $37-48$, mai/ago 2002.

BEZERRA FILHO, J. G; KERR LRFS; MINA D. L; BARRETO M. L. Distribuição espacial da taxa de mortalidade infantil e principais determinantes no ceará, Brasil, no período 2000-2002. Cad. Saúde Pública, v. 23, n. 5, p. 1173-1185. 2007

GUIMARÃES, M. J. B; MARQUES, N. M; MELO FILHO, D. A; SZWARCWALD, C. L. Condição de vida e mortalidade infantil: diferenciais intra-urbanos no Recife, Pernambuco, Brasil. Cad. Saúde Pública, v. 19 n. 5, p. 1413-1424, 2003.

SCHWARZ, J; MARCUS, A. Mortality and Air Pollution in London: A Time Series Analysis. American Journal of Epidemiology, n. 105, 1273-1281, 1990.

VERMELHO L. L; COSTAA. J. L; KALE, P. L. Indicadores de Saúde. In: MEDRONHO, R; CARVALHO,
D. M; BLOCH, K. V; BAGGIO, R; WERNWCK, G. L. (Ed.). Epidemiologia. Rio de Janeiro: Atheneu, 2006. p. 33-55.

CUNHA, T. N; QUINTANILHA, J. A; SILVA, N. N. Amostra mestra e geoprocessamento: tecnologias para inquéritos domiciliares. São Paulo-SP. Rev. de Saúde Pública, v. 37, p. 494-502, 2003.

BARCELLOS, C; BASTOS, F. I. Geoprocessamento, ambiente e saúde: uma união possível? Cad. Saúde Pública, v. 12, p. 389-397, 1996,

IBEGE - INSTITUTO BRASILEIRO DE GEOGRAFIA E ESTATÍSTICA. Censo 2000.

PINTAUDI, M. S. (Org.) VI Seminário Internacional da Unidade Temática de Desenvolvimento Urbano, da Rede de Mercocidades. 1.ed. Buenos Aires: [S. I.] jul. 2003.

AKERMAN, M; CAMPANARIO, P; MAIA, P. B; Saúde e meio ambiente: análise de diferenciais intra-urbanos, Município de São Paulo, Brasil. Rev. Saúde Pública, v. 28, p. 320-355, 1996.

LANDIM, P. M. B. Análise estatística de dados geológicos.2. ed. São Paulo: UNESP, 2003. 253 p.

NETO, P. L. O. C. Estatística. São Paulo: Edgard Blücher, 2002.

BARROS, A. J. D; MATIJASEVICH, A; SANTOS, I. S; ALBERNAZ, E. P; VICTORA, C. G. Neonatal mortality: description and effect of hospital of birth after risk adjustment. Rev. Saúde Pública, v. 42, n. 1, p. 1-9, 2008. 
BENATTI, R. M. Tendência da desigualdade em mortalidade infantil na cidade de Porto Alegre, RS. 2003, 79 f. (Dissertação Mestrado) Faculdade de Medicina da Universidade Federal do Rio Grande do Sul/ Programa de Pós-Graduação em Ciências Médicas: Pediatria. Porto Alegre, 2003.

FISCHER, T. K; LIMA, D; ROSA, R; OSÓRIO, D; BOING, A. F. A mortalidade infantil no brasil: série histórica entre 1994-2004 e associação com indicadores socioeconômicos em municípios de médio e grande porte. Medicina, Ribeirão Preto, v. 40, n. 4, p. 559-566, out/dez 2007. 